\title{
Cryo, Cryo-etch and Tandem Cryo-HRSEM Correlated with Cryo-STEM of Elastin-Mimetic Block Copolymers
}

\author{
E. R. Wright*, V. P. Conticello**, R. P. Apkarian***
}

*Department of Materials Science \& Engineering, University of Southern California, 3651 Watt Way - VHE 510, Los Angeles, CA 90089

** Department of Chemistry, Emory University, 1515 Pierce Drive, Atlanta, GA 30322

***Integrated Microscopy \& Microanalytical Facility, Department of Chemistry, Emory University, 1521 Pierce Drive, Atlanta, GA 30322

Genetic engineering of protein-based materials provides a means for the production of a diverse array of biomaterials. In order to understand some of the basic interactions of materials with biological organisms, the outer topology of the substance must be elucidated. Cryo-HRSEM methods provide a simple and accurate approach for characterization of frozen-hydrated specimens. In this study we focus on the morphological characterization of elastin-mimetic micelles and hydrogels.

Elastin-mimetic block copolymers $\mathbf{1}$ and $\mathbf{2}$ were produced via protein hyper-expression utilizing $\mathrm{pET}$ 24 a plasmid derivatives (Novagen; Madison, WI) and E. coli strain BL21-Gold(DE3) (Stratagene; La Jolla, CA) as the bacterial host [1]. The genes coding for the two polypeptides were synthesized by a revised gene concatamerization protocol $[2,3]$. Bulk samples were prepared as 20 to $25 \%$ protein solutions in sterile $\mathrm{ddH}_{2} \mathrm{O}$. The solutions were pipetted into 3-mm gold planchets (Balzers BU 012 130T), which had been preequilibrated to $4^{\circ} \mathrm{C}$. The temperature of the specimen was raised to between $20^{\circ}$ and $25^{\circ} \mathrm{C}$ and allowed to stabilize. The gelled protein samples were plunge-frozen in liquid ethane at its melting point $\left(-183^{\circ} \mathrm{C}\right)$ or high pressure frozen (Balzers HPM 010); the samples were stored in liquid nitrogen $\left(\mathrm{LN}_{2}\right)[4,5]$. Dilute samples were prepared as 1 to $3 \mathrm{mg} / \mathrm{mL}$ protein solutions in sterile $\mathrm{ddH}_{2} \mathrm{O}$. The solutions were deposited on the surface of lacey carbon coated 300 mesh copper grids, which had been preequilibrated to $25^{\circ} \mathrm{C}$. The solution was allowed to settle on the grid for 60 seconds and excess was wicked away with filter paper. The grids were plunge-frozen in ethane and stored in $\mathrm{LN}_{2}$. A sample, either planchet or grid, was mounted on the precooled Gatan CT-3500 cryo-stage held in the cryo-preparation chamber. The specimen was fractured (planchets) with a prechilled blade and/or washed with $\mathrm{LN}_{2}$. The shutters of the stage were closed and the cryostage was transferred to the Denton DV-602 Cr coater. Specimens were either sputter coated with 1 to $2 \mathrm{~nm}$ of $\mathrm{Cr}$ film or cryo-etched and subsequently coated. The stage shutters were then closed and the stage was transferred to the upper stage of the DS-130 FESEM operated at $25 \mathrm{kV}$.

Three complimentary methods were employed to elucidate the structure of the elastin-mimetic block copolymers as they self-assembled into discrete structures. At high protein concentrations, microscopic phase separation of the protein blocks formed thermoplastic elastomer hydrogels above a lower critical solution temperature $T_{t}$ (Fig. 1a-1d). Initial observations with cryo-HRSEM demonstrated the fairly uniform dispersion of micelles throughout the lakes of vitreous ice (Fig. 1a and 1c). Cryo-etch experiments provided a means for the removal of excess unbound water from the protein hydrogels (Fig. 1b and 1d). This provided information on how the hydrogels were organized from the aggregation of micelles in solution above the $T_{t}$. Tandem cryo-HRSEM correlated with cryo-STEM of dilute aqueous protein solutions afforded data concerning short-range aggregation 
patterns and the mass density of the micelles and micellar clusters (Fig. 1e and 1f). The cryoHRSEM/STEM methods employed enabled us to characterize the morphological features of the elastin-mimetic materials without artifacts that would be incurred with conventional specimen preparation.

References

[1] H. Daniell et al., Methods Mol. Biol. 63 (1997) 359.

[2] R.A. McMillan et al., Macromolecules 32 (1999) 3643.

[3] E.R. Wright et al., Adv. Funct. Mater. 12 (2002) 149.

[4] K.P. Ryan et al., J. Microsc. 145 (1987) 89.

[5] R.P. Apkarian, Acta Microscopia 1 (2000) 20.

[6] This research was supported in part by NASA grant NAG8-1579 (VPC) and NIH grant RR14717-01A1 (RPA).

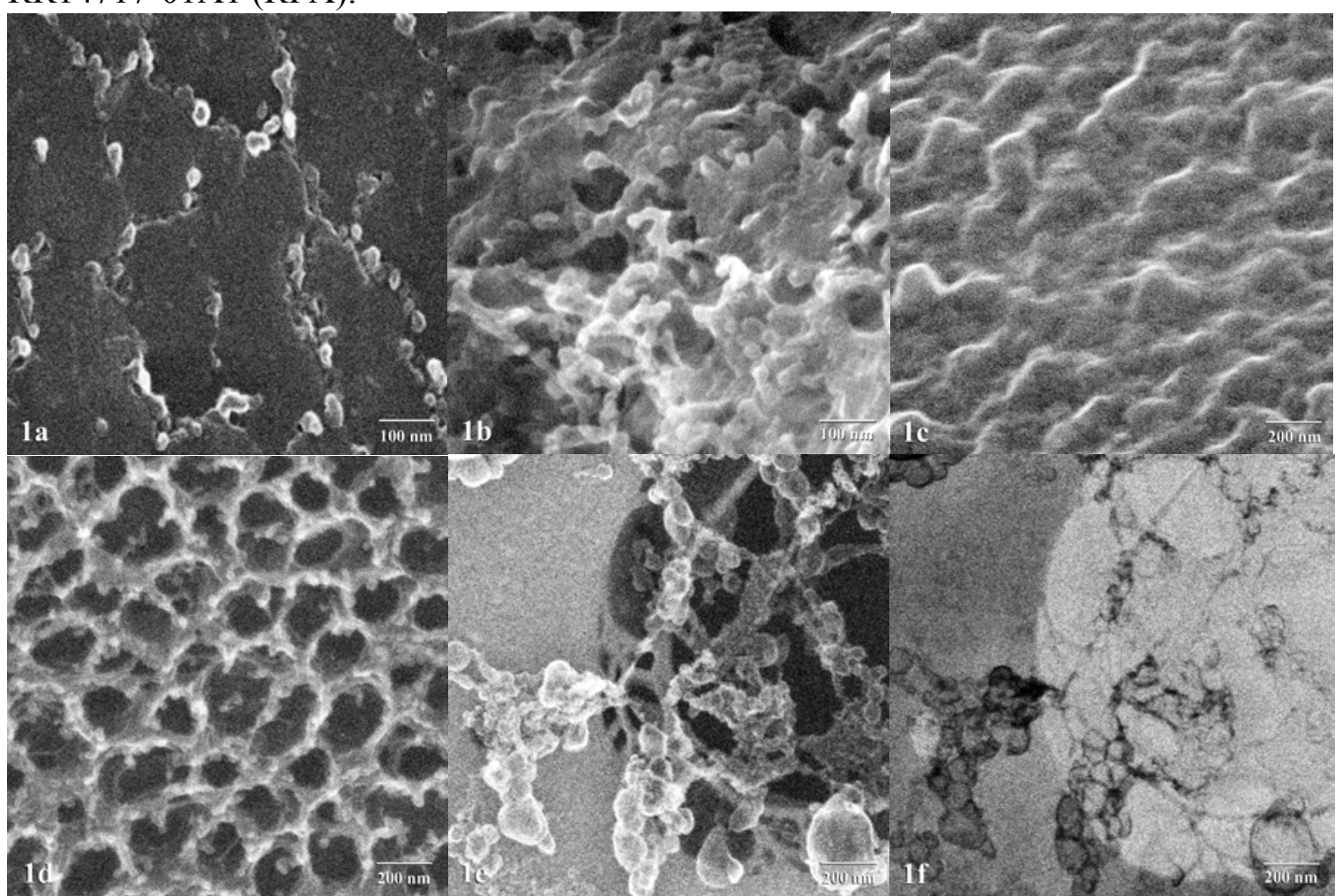

FIG. 1. Cryo, cryo-etch and tandem cryo-HRSEM correlated with cryo-STEM of elastin-mimetic block copolymers. (a) cryo-HRSEM of 1 (25\%), (b) cryo-etch HRSEM of 1 (25\%), (c) cryoHRSEM of 2 (25\%), (d) cryo-etch HRSEM of 2 (25\%), (e) cryo-HRSEM of 1 (2 mg/mL), (f) cryoSTEM of $1(2 \mathrm{mg} / \mathrm{mL})$.

TABLE 1: Amino acid sequence of protein-based block copolymers 1 and $\mathbf{2}$ derived from elastinmimetic protein sequences.

$\left\{\right.$ VPAVG $\left[(\mathrm{IPAVG})_{4}(\mathrm{VPAVG})_{16} \mathrm{IPAVG}\right\}-[\mathrm{X}]-\left\{\mathrm{VPAVG}\left[(\mathrm{IPAVG})_{4}(\mathrm{VPAVG})\right]_{16} \mathrm{IPAVG}\right\}$

1: $[\mathrm{X}]=\mathrm{VPGVG}\left[(\mathrm{VPGVG})_{2} \mathrm{VPGEG}(\mathrm{VPGVG})_{2}\right]_{30} \mathrm{VPGVG}$

2: $[\mathrm{X}]=\mathrm{VPGVG}\left[(\mathrm{VPGVG})_{2} \mathrm{VPGEG}(\mathrm{VPGVG})_{2}\right]_{48} \mathrm{VPGVG}$

A: alanine; E: glutamic acid; G: glycine; I: isoleucine; P: proline; V: valine 